\title{
Frequência de disfagia orofaríngea em idosos institucionalizados: uma revisão integrativa
}

\section{Oropharyngeal dysphagia frequency in older adults living in nursing homes: an integrative review}

\begin{abstract}
Descritores
Transtornos de Deglutição Deglutição Envelhecimento Idoso Instituição de Longa Permanência para
\end{abstract} Idosos

Keywords

Swallowing Disorders Deglutition Aging Aged Homes for the Aged

Endereço para correspondência: Leandro Pernambuco

Departamento de Fonoaudiologia, Universidade Federal da Paraíba UFPB

Cidade Universitária, João Pessoa

(PB), Brasil, CEP: 58051-900.

E-mail: leandroapernambuco@gmail. com

Recebido em: Maio 28, 2020

Aceito em: Julho 13, 2020

\section{RESUMO}

Objetivo: Sintetizar o estado do conhecimento científico sobre a frequência de disfagia orofaríngea em idosos institucionalizados. Estratégia de pesquisa: A pergunta de pesquisa foi formulada de acordo com a estratégia PECO e a busca foi realizada nas bases de dados Pubmed/Medline, Web of Science, Scopus, LILACS e SciELO, utilizando descritores e termos livres específicos. Critérios de seleção: Artigos sem restrição de tempo ou idioma, que relatassem a frequência de disfagia orofaríngea em idosos institucionalizados e o critério utilizado para diagnóstico. Análise dos dados: Foram analisadas as características da população, conceito de "disfagia orofaríngea", métodos para identificação do desfecho e a frequência de disfagia orofaríngea. A avaliação da qualidade metodológica dos artigos seguiu os critérios do Strengthening the Reporting of Observational Studies in Epidemiology (STROBE). Resultados: Foram incluídos quinze artigos. Houve grande variabilidade quanto ao tamanho da amostra, com predomínio de idosos longevos do sexo feminino. O conceito de disfagia, quando mencionado, foi heterogêneo. Os critérios diagnósticos foram diversos e compostos, em sua maioria, por resultados de questionários ou testes clínicos. Nenhum estudo utilizou exames instrumentais. A frequência de disfagia orofaríngea na população estudada oscilou entre $5.4 \%$ e $83.7 \%$, sendo mais elevada nos estudos que utilizaram testes clínicos, porém, com intervalos de confiança mais precisos naqueles que usaram questionários e amostras maiores. Conclusão: A frequência de disfagia orofaríngea em idosos institucionalizados possui ampla variabilidade. As discrepâncias metodológicas entre os estudos comprometem a confiabilidade das estimativas de frequência e apontam a necessidade de pesquisas com critérios metodológicos mais bem definidos e padronizados.

\begin{abstract}
Purpose: To synthesize the scientific knowledge on the frequency of oropharyngeal dysphagia in older adults living in nursing homes. Research strategies: The study question followed the PECO strategy and the search was performed in the Pubmed/Medline, Web of Science, Scopus, LILACS and SciELO databases, using keywords and specific free terms. Selection criteria: articles with no time or language restrictions that reported the frequency of oropharyngeal dysphagia in older adults living in nursing homes and the diagnostic criteria. Data analysis: it was analyzed the population characteristics, the concept of "oropharyngeal dysphagia", the methods for identifying the outcome and the frequency of oropharyngeal dysphagia. The evaluation of the methodological quality of the articles followed the criteria of Strengthening the Reporting of Observational Studies in Epidemiology (STROBE). Results: Fifteen articles were included. There was great variability in relation to the sample size, with a predominance of longevous old women. The concept of dysphagia, when mentioned, was heterogeneous. Diagnostic criteria were diverse and mostly comprised of questionnaires or clinical trials results. No studies used instrumental tests. The frequency of oropharyngeal dysphagia in the studied population ranged from $5.4 \%$ to $83.7 \%$, being higher in studies that used clinical tests, but with greater precision of confidence intervals in studies that used questionnaires and large sample size. Conclusion: The frequency of oropharyngeal dysphagia in older adults living in nursing homes has wide variability. Methodological discrepancies among studies compromise the reliability of frequency estimates and highlight the need for research with better defined and standardized methodological criteria.
\end{abstract}

Trabalho realizado no Programa Associado de Pós-graduação em Fonoaudiologia, Universidade Federal da Paraíba - UFPB - João Pessoa (PB), Brasil.

${ }^{1}$ Programa Associado de Pós-graduação em Fonoaudiologia, Universidade Federal da Paraíba - UFPB - João Pessoa (PB), Brasil.

${ }^{2}$ Programa de Pós-graduação em Saúde Coletiva, Universidade Federal do Rio Grande do Norte - UFRN - Natal $(\mathrm{RN})$, Brasil.

Programa de Pós-graduação em Modelos de Decisão e Saúde, Universidade Federal da Paraíba - UFPB - João Pessoa (PB), Brasil.

${ }^{4}$ Departamento de Fonoaudiologia, Universidade Federal da Paraíba - UFPB - João Pessoa (PB), Brasil.

Fonte de financiamento: nada a declarar.

Conflito de interesses: nada a declarar. 


\section{INTRODUÇÃO}

A proporção de pessoas idosas no mundo está aumentando e o envelhecimento populacional é um dos fenômenos mais significativos do século XXI ${ }^{(1,2)}$. Em 2018, a quantidade de idosos ultrapassou a quantidade de crianças com menos de cinco anos de idade ${ }^{(1)}$. Estima-se que a proporção de idosos no mundo chegue a $11.7 \%$ em 2030, $15.9 \%$ em 2050 e atinja $22.6 \%$ em $2100^{(1,2)}$. O número de pessoas acima de 80 anos está crescendo ainda mais rápido, já que em 1990 havia apenas 54 milhões de pessoas com 80 anos ou mais no mundo e em 2019 esse número quase triplicou para 143 milhões $^{(1)}$. No Brasil, de acordo com o Censo 2010, existem mais de 20 milhões de pessoas com 60 anos ou mais, que representam $10.8 \%$ do total da população ${ }^{(3)}$. Com isso, a população brasileira desenha uma estrutura etária padrão de envelhecimento, em que há o estreitamento da base da pirâmide etária, devido a diminuição dos níveis de fecundidade e o alargamento do topo, em decorrência da redução dos níveis de mortalidade ${ }^{(3)}$.

O envelhecimento está inevitavelmente associado ao declínio funcional, caracterizado por um comprometimento na realização das atividades de vida diária, incluindo as limitações relacionadas à alimentação ${ }^{(4)}$. O contexto da alimentação tem importante papel cultural na sociedade e na expressão da identidade pessoal do idoso ${ }^{(5)}$. Como está atrelada ao ato de se alimentar, a deglutição garante prazer e está inserida num contexto de relações sociais e afetivas ${ }^{(6)}$. Neste cenário, a função da deglutição, é extremamente essencial, desde a apresentação visual do alimento até a ingestão(7).

A partir dos 60 anos, os indivíduos apresentam maior vulnerabilidade para variados tipos de doenças e incapacidades, incluindo maior risco para desenvolver alterações de deglutição devido as mudanças naturais que ocorrem na dinâmica dessa função, e o surgimento mais frequente de doenças específicas ou quadros degenerativos que podem interferir nessa função, a exemplo do acidente vascular encefálico e as demências ${ }^{(8)}$.

As principais características na dificuldade da deglutição nos idosos incluem diminuição da sensibilidade gustativa e da percepção da viscosidade do alimento, enfraquecimento da musculatura lingual para a propulsão do bolo alimentar, lentificação no início da resposta faríngea para deglutir, lentificação na elevação do hioide e alterações na eficiência da deglutição, por presença de resíduos orofaríngeos, com sinais de penetração/aspiração laringotraqueal ${ }^{(9-11)}$. Além dos aspectos anatomofisiológicos, também devem ser consideradas as alterações no prazer ao alimentar-se e o impacto negativo na socialização ${ }^{(12)}$.

Todo esse cenário pode tornar a deglutição do idoso menos eficiente, contribuindo para o aparecimento da disfagia orofaríngea $^{(13)}$. A deglutição consiste no ato de conduzir o alimento da cavidade oral em direção ao estômago no intuito de manter o estado nutricional e hídrico, sem permitir a entrada de material nas vias aéreas ${ }^{(14)}$. Quando surgem transtornos nesse processo e a ele são adicionadas alterações neurológicas, anatômicas ou fisiológicas de ordem patológica, surge a disfagia orofaríngea ${ }^{(15)}$.

Observa-se que a ocorrência de disfagia orofaríngea varia de acordo com o grupo estudado e pode alcançar índices de $70 \%$ a $90 \%$ nas populações mais envelhecidas ${ }^{(16)}$, constituindo-se um dos grandes problemas de saúde presentes em idosos institucionalizados ${ }^{(17,18)}$. Nos Estados Unidos, estima-se que $40 \%$ a $60 \%$ dos idosos residentes em instituições de longa permanência (ILPI) apresentam evidências clínicas de disfagia orofaríngea comparado a $13 \%$ a $33 \%$ dos idosos não institucionalizados ${ }^{(19)}$.

Alguns estudos verificaram que a disfagia orofaríngea em residentes de ILPI está frequentemente relacionada a Acidente Vascular Encefálico (AVE), dependência, demência avançada, higiene oral precária, múltiplos diagnósticos e fatores ambientais $^{(20-22)}$. Some-se a isso a sarcopenia, síndrome geriátrica clínica à qual a disfagia pode estar associada ${ }^{(23)}$, caracterizada pelo declínio da função física devido à diminuição da força muscular resultante da perda de massa muscular esquelética ${ }^{(24)}$. Ademais, os problemas vivenciados pelos idosos durante a refeição não se restringem aos sinais disfágicos, incluem alterações cognitivas, comportamentais, físicas e ambientais que, conjuntamente, predispõem o idoso a uma mudança na dinâmica alimentar e riscos de pneumonia broncoaspirativa, desnutrição e desidratação ${ }^{(25)}$.

A disfagia compromete a segurança e eficiência da deglutição, causando, respectivamente, pneumonia por broncoaspiração e danos às necessidades nutricionais e hídricas do idoso ${ }^{(26)}$. Dessa forma, compreender a frequência da disfagia orofaríngea em idosos institucionalizados permite conhecer o impacto dessa condição na saúde do idoso, e ajuda a gerenciar os problemas de alimentação e deglutição desta população, a fim de determinar intervenções individuais e coletivas, fonoaudiológicas e interdisciplinares, visando o bem-estar de cada idoso e à redução de custos em saúde(27).

Existe uma notória variabilidade na literatura em relação aos registros da frequência de disfagia orofaríngea na população idosa institucionalizada, o que compromete o dimensionamento mais preciso da magnitude dessa condição de saúde em idosos residentes em ILPI. Compreender esse cenário é importante para o adequado planejamento e execução das ações de cuidado e atenção à disfagia orofaríngea voltadas a essa população.

\section{OBJETIVO}

O objetivo deste estudo foi sintetizar o estado do conhecimento científico sobre a frequência de disfagia orofaríngea em idosos institucionalizados.

\section{ESTRATÉGIA DE PESQUISA}

Este estudo trata-se de uma revisão da literatura do tipo integrativa, portanto, não houve necessidade de submetê-lo à avaliação do Comitê de Ética e Pesquisa em Seres Humanos da instituição. Os procedimentos metodológicos seguiram as etapas recomendadas na literatura para esse tipo de revisão ${ }^{(28)}$.

Inicialmente, a pergunta de pesquisa foi formulada seguindo a estratégia PECO, acrônimo que representa os elementos Paciente, Exposição, Comparação e Resultados, respectivamente. $\mathrm{O}$ primeiro elemento da estratégia $(\mathrm{P})$ correspondeu a idosos residentes em instituições de longa permanência; o segundo elemento (E) correspondeu à "disfagia orofaríngea"; o terceiro elemento (C), equivalente ao "grupo de comparação", não foi 
aplicado nesta revisão; e o quarto elemento $(\mathrm{O})$ foi a frequência de disfagia orofaríngea. Diante disso, a questão da pesquisa foi definida da seguinte forma: "Qual a frequência de disfagia orofaríngea em idosos residentes em ILPI?”.

A busca incluiu as bases de dados eletrônicas Medline/PubMed, Web of Science, Scopus, SciELO e Lilacs. As estratégias de busca incluíram combinações entre descritores do Medical Subjects Headings (MeSH) e termos livres considerados relevantes para a pesquisa (Apêndice $\mathrm{A}$ ).

\section{CRITÉRIOS DE SELEÇÃO}

Foram aplicados os seguintes critérios de inclusão: artigos que investigaram a frequência de disfagia orofaríngea em idosos institucionalizados, sem restrição de tempo ou idioma. Os critérios de exclusão aplicados foram: artigos de revisão de qualquer tipo, editoriais, cartas ao editor, anais de congresso, teses e dissertações. Foram também excluídos artigos que não descreveram o critério diagnóstico para determinar a presença de disfagia orofaríngea e aqueles que incluíram idosos institucionalizados na amostra, mas não apresentaram a frequência de disfagia orofaríngea nesse grupo populacional.

\section{ANÁLISE DOS DADOS}

Os estudos foram avaliados independentemente por dois pesquisadores. Depois, eles compararam suas análises e as divergências foram resolvidas por um terceiro avaliador. Após a identificação dos artigos nas bases de dados e filtragem para exclusão de duplicados, executou-se a triagem, na qual foram lidos os respectivos títulos e resumos e excluídos aqueles que não atendessem aos critérios de inclusão. Na fase de elegibilidade, os demais artigos que potencialmente tratavam do assunto foram submetidos a uma revisão de texto completo. Ainda nesta fase, as listas de referências dos artigos foram checadas manualmente e os estudos não identificados anteriormente pela estratégia de busca foram investigados.

Os artigos que atendiam aos critérios de elegibilidade foram submetidos à extração dos seguintes dados para composição da matriz de análise: localização do estudo, população, tamanho da amostra, sexo dos participantes, idade, conclusão do estudo, instrumentos de diagnóstico, conceito de disfagia orofaríngea e frequência de disfagia orofaríngea. Para determinar a precisão das estimativas de frequência em cada estudo, os autores desta revisão calcularam o intervalo de confiança (IC) e a margem de erro do IC.

A avaliação da qualidade metodológica dos estudos seguiu os critérios do Strengthening the Reporting of Observational Studies in Epidemiology (STROBE) ${ }^{(29,30)}$.

\section{RESULTADOS}

Um total de 1.361 artigos foram localizados inicialmente. Após a aplicação dos filtros de elegibilidade, a amostra final foi composta por quinze artigos. O processo de seleção é mostrado na Figura 1.

A matriz de análise com as características dos estudos que atenderam aos critérios de elegibilidade ${ }^{(12,18,31-43)}$ está apresentada no Quadro 1.

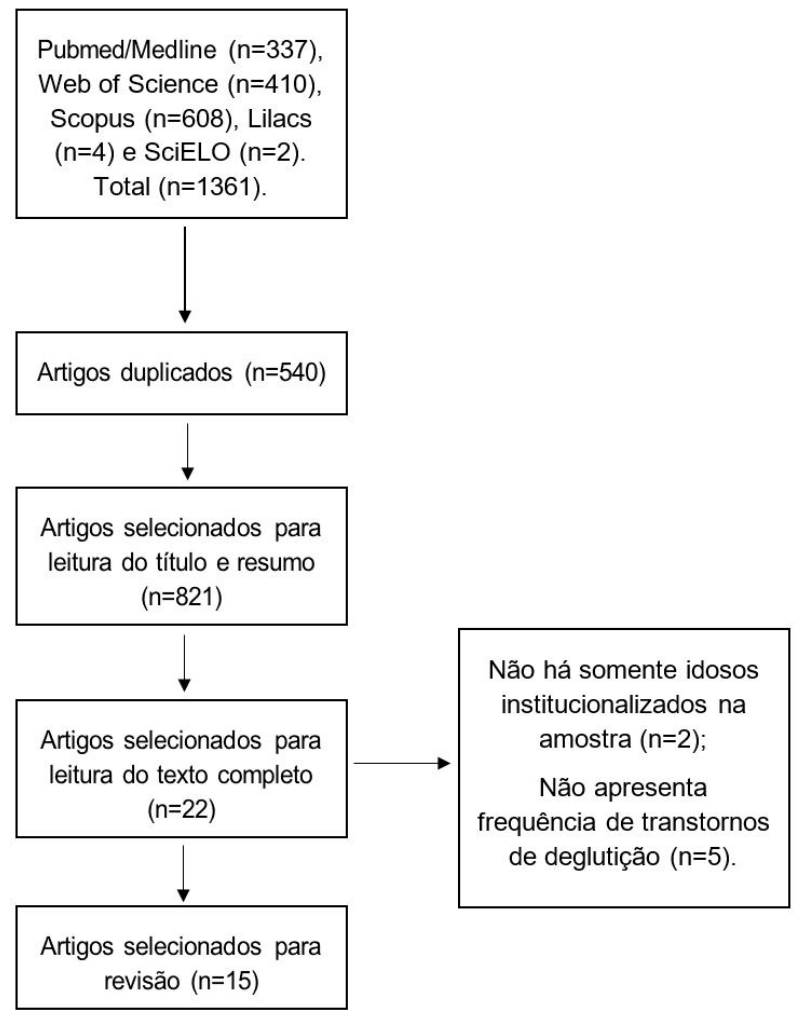

Figura 1. Fluxograma da seleção dos artigos 
Quadro 1. Matriz de análise com as características dos estudos incluídos na revisão integrativa

\begin{tabular}{|c|c|c|c|c|c|c|}
\hline $\begin{array}{c}\text { Autor, ano e } \\
\text { local (crescente) }\end{array}$ & Amostra & Sexo & Idade & $\begin{array}{c}\text { Conceito de disfagia } \\
\text { orofaríngea }\end{array}$ & Instrumento & $\begin{array}{c}\text { Frequência de } \\
\text { disfagia orofaríngea* }\end{array}$ \\
\hline \multirow[b]{3}{*}{$\begin{array}{l}\text { Roque et al., } \\
\text { 2010, Brasil(31) }\end{array}$} & \multirow[b]{3}{*}{$\begin{array}{l}30 \text { idosas } \\
\text { residentes em } \\
\text { uma ILPI }\end{array}$} & \multirow[b]{3}{*}{$\begin{array}{l}100 \% \text { sexo } \\
\text { feminino }\end{array}$} & \multirow[b]{3}{*}{$\begin{array}{l}83,7 \pm 10,7 \\
\text { anos }\end{array}$} & \multirow[b]{3}{*}{ Não define claramente } & \multirow{3}{*}{$\begin{array}{c}\text { Avaliadas características } \\
\text { da refeição; ambiente; } \\
\text { aspectos atitudinais } \\
\text { e comportamentais; } \\
\text { dependência alimentar } \\
\text { e assistência requerida/ } \\
\text { provida; aspectos } \\
\text { bucais; problemas de } \\
\text { deglutição e dificuldade } \\
\text { respiratória }\end{array}$} & $23,30 \%$ \\
\hline & & & & & & (IC95\%: 8,17-38,42) \\
\hline & & & & & & $\begin{array}{c}\text { Margem de erro da } \\
\text { estimativa }=30,25 \%\end{array}$ \\
\hline \multirow{3}{*}{$\begin{array}{l}\text { Camacho et al., } \\
\text { 2011, } \\
\text { Espanha(32) }^{(32)}\end{array}$} & \multirow{3}{*}{$\begin{array}{l}1.777 \text { idosos } \\
\text { residentes de } \\
66 \text { ILPI }\end{array}$} & \multirow{3}{*}{$\begin{array}{l}80,8 \% \text { sexo } \\
\text { feminino }\end{array}$} & \multirow{3}{*}{$\begin{array}{c}>85 \text { anos } \\
52,08 \% \text {, de } \\
75 \text { a } 84 \text { anos } \\
36,45 \% \text { e de } \\
65 \text { a } 74 \text { anos } \\
11,45 \%\end{array}$} & \multirow{3}{*}{$\begin{array}{l}\text { Dificuldade em engolir } \\
\text { alimentos ou bebidas }\end{array}$} & \multirow{3}{*}{$\begin{array}{c}\text { Questionário preparado } \\
\text { pela Geriatric Home } \\
\text { Care Unit (UADG) } \\
\text { aconselhado pela Nestlé } \\
\text { Nutrition }\end{array}$} & $5,40 \%$ \\
\hline & & & & & & (IC95\%: 4.34-6,45) \\
\hline & & & & & & $\begin{array}{l}\text { Margem de erro da } \\
\text { estimativa }=2.10 \%\end{array}$ \\
\hline \multirow{3}{*}{$\begin{array}{c}\text { Ferrero } \\
\text { López et al., } \\
2012, \\
\text { Espanha }^{(33)}\end{array}$} & \multirow{3}{*}{$\begin{array}{l}40 \text { idosos } \\
\text { residentes em } \\
\text { cinco ILPI }\end{array}$} & \multirow{3}{*}{$\begin{array}{l}72,5 \% \text { sexo } \\
\text { feminino }\end{array}$} & \multirow{3}{*}{$\begin{array}{l}83,7 \pm 6,3 \\
\text { anos }\end{array}$} & \multirow{3}{*}{$\begin{array}{c}\text { Alterações tanto } \\
\text { estruturais quanto } \\
\text { funcionais da deglutição }\end{array}$} & \multirow{3}{*}{$\begin{array}{l}\text { Método de Exploração } \\
\text { Clínica Volume- } \\
\text { Viscosidade para a } \\
\text { detecção da Disfagia } \\
\text { Orofaríngea (MECVV) }\end{array}$} & $65 \%$ \\
\hline & & & & & & (IC95\%: 50,21-79,78) \\
\hline & & & & & & $\begin{array}{c}\text { Margem de erro da } \\
\text { estimativa }=29.56 \%\end{array}$ \\
\hline \multirow{3}{*}{$\begin{array}{l}\text { Bomfim et al., } \\
\text { 2013, Brasil(12) }\end{array}$} & \multirow{3}{*}{$\begin{array}{l}30 \text { idosas } \\
\text { residentes em } \\
\text { uma ILPI }\end{array}$} & \multirow{3}{*}{$\begin{array}{l}100 \% \text { sexo } \\
\text { feminino }\end{array}$} & \multirow{3}{*}{$\begin{array}{l}83,7 \pm 10,5 \\
\text { anos }\end{array}$} & \multirow{3}{*}{$\begin{array}{c}\text { Alterações da dinâmica } \\
\text { alimentar }\end{array}$} & \multirow{3}{*}{$\begin{array}{l}\text { Baseado no protocolo } \\
\text { fonoaudiológico de } \\
\text { avaliação do risco para } \\
\text { disfagia - PARD }\end{array}$} & $63,30 \%$ \\
\hline & & & & & & (IC95\%: 46,05-80,54) \\
\hline & & & & & & $\begin{array}{c}\text { Margem de erro da } \\
\text { estimativa }=34,49 \%\end{array}$ \\
\hline \multirow{3}{*}{$\begin{array}{c}\text { Park et al., } \\
\text { 2013, Coréia do } \\
\text { Sul( }^{(34)}\end{array}$} & \multirow{3}{*}{$\begin{array}{l}395 \text { idosos } \\
\text { residentes em } \\
\text { duas ILPI }\end{array}$} & \multirow{3}{*}{$\begin{array}{l}76,5 \% \text { sexo } \\
\text { feminino }\end{array}$} & \multirow{3}{*}{$\begin{array}{l}80.7 \pm 8.0 \\
\text { anos }\end{array}$} & \multirow{3}{*}{$\begin{array}{c}\text { Comprometimento na } \\
\text { deglutição }\end{array}$} & \multirow{3}{*}{$\begin{array}{l}\text { Gugging Swallowing } \\
\text { Screen (GUSS) }\end{array}$} & $52,70 \%$ \\
\hline & & & & & & (IC95\%: 47,77-57,62) \\
\hline & & & & & & $\begin{array}{l}\text { Margem de erro da } \\
\text { estimativa }=9,84 \%\end{array}$ \\
\hline \multirow{3}{*}{$\begin{array}{l}\text { Nogueira et al., } \\
2013, \\
\text { Portugal( }^{(35)}\end{array}$} & \multirow{3}{*}{$\begin{array}{l}266 \text { idosos } \\
\text { residentes em } \\
\text { oito ILPI }\end{array}$} & & & & & $38,20 \%$ \\
\hline & & $75 \%$ sexo & $82 \pm 10$ anos & Trata de presbifagia & $\begin{array}{c}3 \text { oz Water Swallow Test } \\
\text { (3OZwst) e Dysphagia }\end{array}$ & (IC95\%: 32,36-44,03) \\
\hline & & feminino & & & Self-Test (DST) & $\begin{array}{c}\text { Margem de erro da } \\
\text { estimativa }=11,67 \%\end{array}$ \\
\hline & & & & Sintoma que se refere & Preenchimento de & $9 \%$ \\
\hline $\begin{array}{l}\text { Van der Maarel- } \\
\text { Wierink et al., }\end{array}$ & $\begin{array}{l}8119 \\
\text { residentes }\end{array}$ & $74 \%$ sexo & $84.0 \pm 7.0$ & $\begin{array}{c}\text { à dificuldade ou } \\
\text { desconforto durante }\end{array}$ & $\begin{array}{c}\text { questionário pelos } \\
\text { cuidadores e resposta }\end{array}$ & (IC95\%: 8,37-9,62) \\
\hline $\begin{array}{c}2014, \\
\text { Holanda }^{(18)}\end{array}$ & $\begin{array}{l}\text { com } \geq 65 \text { anos } \\
\text { de } 119 \text { ILPI }\end{array}$ & feminino & anos & $\begin{array}{l}\text { a progressão do bolo } \\
\text { alimentar da cavidade } \\
\text { oral ao estômago }\end{array}$ & $\begin{array}{c}\text { do residente a pergunta: } \\
\text { "Do you experience } \\
\text { swallowing problems?" }\end{array}$ & $\begin{array}{l}\text { Margem de erro da } \\
\text { estimativa }=1,24 \%\end{array}$ \\
\hline & & & & Indica a existência de & & $69,60 \%$ \\
\hline $\begin{array}{l}\text { Sarabla- } \\
\text { Cobo et al., }\end{array}$ & $\begin{array}{l}2384 \text { idosos } \\
\text { residentes em }\end{array}$ & $73,4 \%$ sexo & $88.7 \pm 6.8$ & $\begin{array}{l}\text { dificuldade ao passar } \\
\text { alimentos ou líquidos da }\end{array}$ & $\begin{array}{l}\text { Eating Assessment lool- } \\
10 \text { (EAT_10) e } 3 \text { ounce }\end{array}$ & (IC95\%: 67,75-71,44) \\
\hline $\begin{array}{l}\text { 2016, } \\
\text { Espanha }^{(36)}\end{array}$ & 12 ILPI & feminino & anos & $\begin{array}{c}\text { boca e do esôfago para } \\
\text { o estômago }\end{array}$ & $\begin{array}{c}\text { Water Swallow Test } \\
\text { (3OZwst) }\end{array}$ & $\begin{array}{l}\text { Margem de erro da } \\
\text { estimativa }=3,69\end{array}$ \\
\hline & & $76,2 \%$ & $\begin{array}{l}87,7 \pm 6,4 \\
\text { anos }\end{array}$ & & & $16,90 \%$ \\
\hline & 236 residentes & $\begin{array}{l}\text { mulneres } \\
\text { no grupo de }\end{array}$ & para o grupo & Distúrbio de qualquer & & (IC95\%: 12,11-21,68) \\
\hline 2017, Japão(37) & $\begin{array}{l}\text { com } \geq 60 \text { anos } \\
\text { de oito ILPIs }\end{array}$ & $\begin{array}{c}\text { dentado e } \\
52,6 \% \text { no } \\
\text { grupo de } \\
\text { desdentados }\end{array}$ & $\begin{array}{l}\text { de dentados } \\
\text { e } 89,0 \pm 7,1 \\
\text { anos para } \\
\text { o grupo de } \\
\text { desdentados }\end{array}$ & $\begin{array}{c}\text { parte do processo de } \\
\text { deglutição }\end{array}$ & $\begin{array}{l}\text { Mioditied Vater SWallow } \\
\text { Test (MWST) }\end{array}$ & $\begin{array}{l}\text { Margem de erro da } \\
\text { estimativa }=9,56 \%\end{array}$ \\
\hline
\end{tabular}

*Intervalo de confiança e margem de erro da estimativa calculados pelos autores deste estudo

Legenda: IC = Intervalo de Confiança; ILPI = Instituição de Longa Permanência para Idosos 
Quadro 1. Continuação...

\begin{tabular}{|c|c|c|c|c|c|c|}
\hline $\begin{array}{c}\text { Autor, ano e } \\
\text { local (crescente) }\end{array}$ & Amostra & Sexo & Idade & $\begin{array}{c}\text { Conceito de disfagia } \\
\text { orofaríngea }\end{array}$ & Instrumento & $\begin{array}{c}\text { Frequência de } \\
\text { disfagia orofaríngea* }\end{array}$ \\
\hline \multirow{3}{*}{$\begin{array}{l}\text { Streicher et al., } \\
\text { 2017, Europa } \\
\text { e América do } \\
\text { Norte }^{(38)}\end{array}$} & \multirow{3}{*}{$\begin{array}{c}23.549 \\
\text { residentes } \\
\text { com } \geq 65 \text { anos } \\
\text { de } 926 \text { ILPIs }\end{array}$} & \multirow{3}{*}{$\begin{array}{l}75,7 \% \text { sexo } \\
\text { feminino }\end{array}$} & \multirow{3}{*}{$\begin{array}{l}\text { Entre } 79-90 \\
\text { anos }\end{array}$} & \multirow{3}{*}{$\begin{array}{l}\text { Ingestão prejudicada } \\
\text { e potencialmente } \\
\text { insegura de líquidos e/ou } \\
\text { alimentos sólidos }\end{array}$} & \multirow{3}{*}{$\begin{array}{c}\text { Informações da equipe } \\
\text { local e prontuário médico } \\
\text { do residente }\end{array}$} & $13,40 \%$ \\
\hline & & & & & & (IC95\%: 12,96-13,83) \\
\hline & & & & & & $\begin{array}{l}\text { Margem de erro da } \\
\text { estimativa }=0,87 \%\end{array}$ \\
\hline \multirow{3}{*}{$\begin{array}{l}\text { Namasivayam- } \\
\text { MacDonald et al., } \\
\text { 2017, Canadá( }{ }^{39)}\end{array}$} & \multirow{3}{*}{$\begin{array}{l}639 \text { idosos } \\
\text { residentes de } \\
32 \text { ILPI }\end{array}$} & \multirow{3}{*}{$\begin{array}{l}69,9 \% \text { sexo } \\
\text { feminino }\end{array}$} & \multirow{3}{*}{$\begin{array}{l}86.8 \pm 7.8 \\
\quad \text { anos }\end{array}$} & \multirow{3}{*}{$\begin{array}{l}\text { Comprometimento na } \\
\text { deglutição }\end{array}$} & \multirow{3}{*}{$\begin{array}{c}\text { Iowa Oral Performance } \\
\text { Instrument e Screening } \\
\text { Tool for Acute Neuro } \\
\text { Dysphagia }\end{array}$} & $59,20 \%$ \\
\hline & & & & & & (IC95\%: 55,38-63,01) \\
\hline & & & & & & $\begin{array}{l}\text { Margem de erro da } \\
\text { estimativa }=7,62 \%\end{array}$ \\
\hline \multirow{3}{*}{$\begin{array}{l}\text { Fernández- } \\
\text { Getino } \\
\text { Sallés, 2018, } \\
\text { Espanha }^{(40)}\end{array}$} & \multirow{3}{*}{$\begin{array}{l}30 \text { indivíduos } \\
\text { com }>80 \text { anos } \\
\text { residentes em } \\
\text { uma ILPI }\end{array}$} & \multirow{3}{*}{$\begin{array}{l}90 \% \text { sexo } \\
\text { feminino }\end{array}$} & \multirow{3}{*}{$\begin{array}{l}89,3 \pm 1,1 \\
\quad \text { anos }\end{array}$} & \multirow{3}{*}{$\begin{array}{l}\text { Diminuição da segurança } \\
\text { e eficácia da deglutição }\end{array}$} & \multirow{3}{*}{$\begin{array}{c}\text { Método de Exploração } \\
\text { Clínica Volume- } \\
\text { Viscosidade para a } \\
\text { detecção da Disfagia } \\
\text { Orofaríngea (MECVV) }\end{array}$} & $41,50 \%$ \\
\hline & & & & & & (IC95\%: 23,86-59,13) \\
\hline & & & & & & $\begin{array}{l}\text { Margem de erro da } \\
\text { estimativa }=35,26\end{array}$ \\
\hline \multirow{3}{*}{$\begin{array}{l}\text { Jukic } \\
\text { Peladic et al., } \\
\text { 2018, Itália }\end{array}$} & \multirow{3}{*}{$\begin{array}{l}1490 \\
\text { residentes } \\
\text { com } \geq 65 \text { anos } \\
\text { residentes em } \\
\quad 31 \text { ILPIs }\end{array}$} & \multirow{3}{*}{$\begin{array}{l}71,5 \% \text { sexo } \\
\text { feminino }\end{array}$} & \multirow{3}{*}{$\begin{array}{l}83,5 \pm 8,1 \\
\quad \text { anos }\end{array}$} & \multirow{3}{*}{$\begin{array}{l}\text { Dificuldade em engolir } \\
\text { líquidos e/ou alimentos } \\
\text { sólidos }\end{array}$} & \multirow{3}{*}{ Avaliação clínica } & $12,80 \%$ \\
\hline & & & & & & (IC95\%: 11,10-14,49) \\
\hline & & & & & & $\begin{array}{l}\text { Margem de erro da } \\
\text { estimativa }=3,39 \%\end{array}$ \\
\hline \multirow[b]{3}{*}{$\begin{array}{c}\text { Huppertz et al., } \\
2018, \\
\text { Holanda }^{(42)}\end{array}$} & \multirow{3}{*}{$\begin{array}{c}6349 \\
\text { residentes } \\
\text { de um lar de } \\
\text { idosos com } \\
\geq 65 \text { anos } \\
\text { internados em } \\
\text { enfermarias } \\
\text { psicogeriátricas } \\
\text { ou somáticas }\end{array}$} & \multirow[b]{3}{*}{$\begin{array}{l}70.12 \text { sexo } \\
\text { feminino }\end{array}$} & \multirow[b]{3}{*}{$\begin{array}{l}84,5 \pm 7,5 \\
\quad \text { anos }\end{array}$} & \multirow[b]{3}{*}{ Não define claramente } & \multirow{3}{*}{$\begin{array}{l}\text { Baseado em duas } \\
\text { questões: "Does the } \\
\text { client have swallowing } \\
\text { problems?" e "Does the } \\
\text { client sneeze or cough } \\
\text { while swallowing food or } \\
\text { liquids?" }\end{array}$} & $12,10 \%$ \\
\hline & & & & & & (IC95\%: 11,29-12,90) \\
\hline & & & & & & $\begin{array}{l}\text { Margem de erro da } \\
\text { estimativa }=1,60 \%\end{array}$ \\
\hline \multirow{3}{*}{$\begin{array}{l}\text { Hoshino et al., } \\
\text { 2020, Japão(43) }\end{array}$} & \multirow{3}{*}{$\begin{array}{c}312 \text { idosos } \\
\text { com } \\
\text { demência } \\
\text { avançada } \\
\text { residentes em } \\
\text { ILPI }\end{array}$} & \multirow{3}{*}{$\begin{array}{l}79,5 \% \text { sexo } \\
\text { feminino }\end{array}$} & \multirow{3}{*}{$\begin{array}{l}85,2 \pm 7,6 \\
\quad \text { anos }\end{array}$} & \multirow{3}{*}{ Não define claramente } & \multirow{3}{*}{$\begin{array}{c}\text { Modified water } \\
\text { swallowing test (MWST) } \\
\text { combinado com ausculta } \\
\text { cervical }\end{array}$} & $83,70 \%$ \\
\hline & & & & & & (IC95\%: 79,60-87,79) \\
\hline & & & & & & $\begin{array}{l}\text { Margem de erro da } \\
\text { estimativa }=8,19 \%\end{array}$ \\
\hline
\end{tabular}

*Intervalo de confiança e margem de erro da estimativa calculados pelos autores deste estudo

Legenda: IC = Intervalo de Confiança; ILPI = Instituição de Longa Permanência para Idosos

Os artigos incluídos foram publicados entre os anos de $2010 \mathrm{e}$ 2020, com destaque à maior concentração de estudos publicados por pesquisadores europeus. As amostras se apresentaram heterogêneas quanto ao número de ILPI participantes e a quantidade de idosos, sendo a menor amostra composta por 30 idosos e a maior por $23.549 \mathrm{em}$ um estudo multicêntrico. Em todos os artigos, a população foi predominantemente do sexo feminino, com média de idade acima de 80 anos.

O conceito de disfagia orofaríngea foi distinto entre os estudos e sem definição explícita em três casos. Nenhum dos métodos descritos nos artigos referiu o uso de exames instrumentais para avaliação e diagnóstico da disfagia orofaríngea. Onze estudos utilizaram a avaliação clínica da deglutição e quatro elaboraram questionários próprios para determinar a presença de disfagia orofaríngea.

A frequência de disfagia orofaríngea variou de forma ampla entre 5.4\% (IC95\%: 4.34-6.45) ${ }^{(32)}$ e $83.7 \%$ (IC95\%: $79.60-87.79)^{(43)}$. As estimativas de frequência foram mais altas em estudos que utilizaram testes clínicos ${ }^{(12,33,34,39,40,43)}$. Entretanto, observou-se intervalos de confiança mais precisos, isto é, com margens de erro mais baixas, em estudos com amostras maiores e que usaram questionários ${ }^{(18,32,38,42)}$.

A qualidade metodológica dos estudos incluídos nesta revisão está apresentada no Quadro 2. De acordo com os critérios do STROBE os itens mais insatisfatórios foram o 12c, 13c, 14b e 22. Nenhum dos quinze estudos atendeu a todos os critérios de avaliação da qualidade metodológica. 


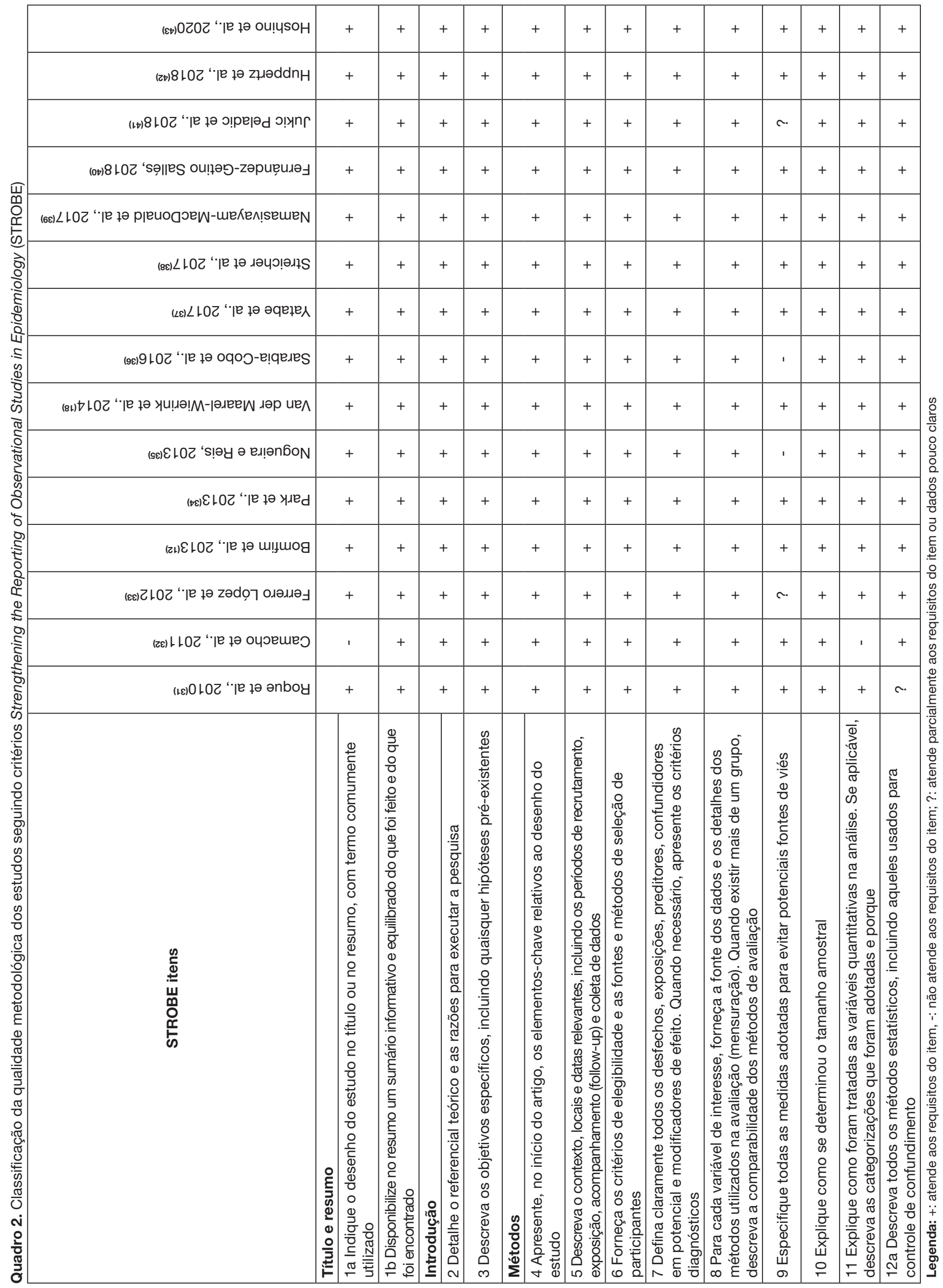




\begin{tabular}{|c|c|c|c|c|c|c|c|c|c|c|c|c|c|}
\hline 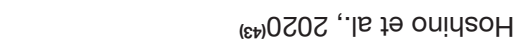 & + & c. & + & + & + & + & + & + & c. & + & + & + & \\
\hline 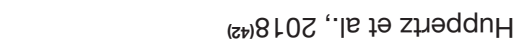 & + & + & + & + & + & c. & ' & + & ' & + & + & + & + \\
\hline 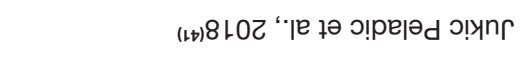 & + & c. & + & + & + & + & ' & + & ' & + & + & + & + \\
\hline 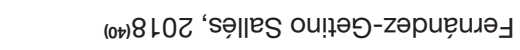 & + & ' & + & + & + & + & ' & + & ' & + & + & + & + \\
\hline 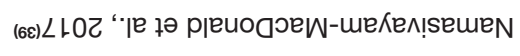 & + & ' & + & + & + & + & ' & + & ' & + & + & + & + \\
\hline 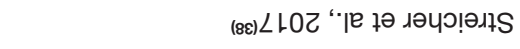 & + & 1 & + & + & + & + & + & + & 1 & + & + & + & + \\
\hline 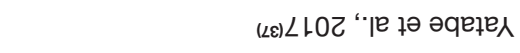 & + & ' & + & + & + & c. & ' & + & ' & + & + & + & + \\
\hline 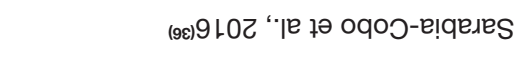 & + & ' & + & + & + & + & ' & + & ' & + & + & + & + \\
\hline 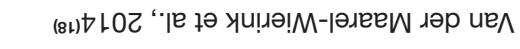 & + & ' & + & + & + & + & ' & + & ' & + & + & + & + \\
\hline (ઘءદ & + & ' & + & ' & + & + & ' & + & ' & + & ' & + & ' \\
\hline 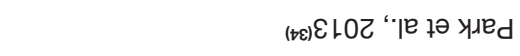 & + & ' & + & + & + & + & + & + & ' & + & + & + & + \\
\hline 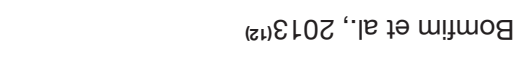 & + & + & + & + & + & + & ' & + & ' & + & + & + & + \\
\hline 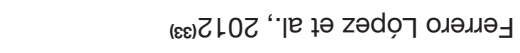 & + & ' & + & + & + & + & ' & + & ' & + & + & + & + \\
\hline 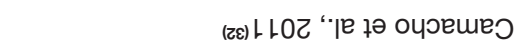 & ' & ' & + & ' & + & ' & ' & + & ' & + & ' & + & ' \\
\hline (เ\&) 0 LOZ ' "| & ' & ' & + & ' & + & ' & 1 & + & + & + & ' & + & ' \\
\hline 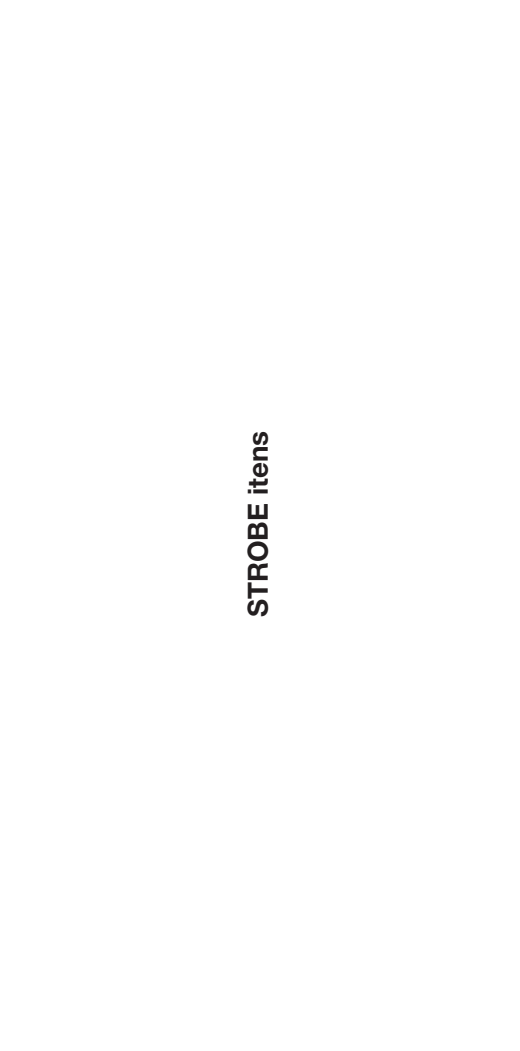 & 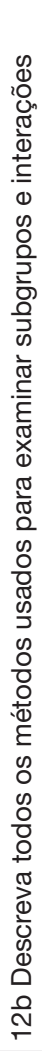 & 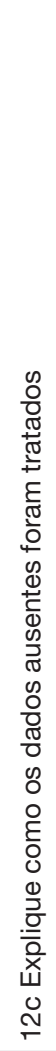 & 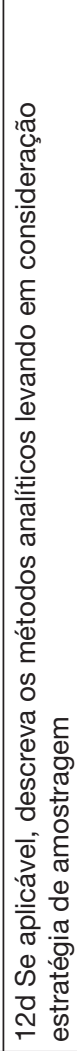 & 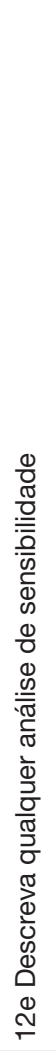 & 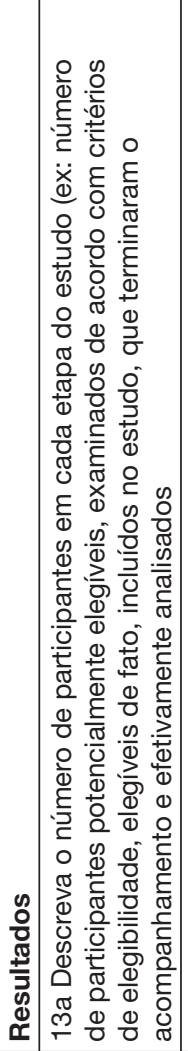 & 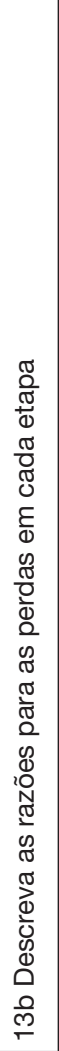 & 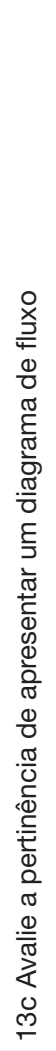 & 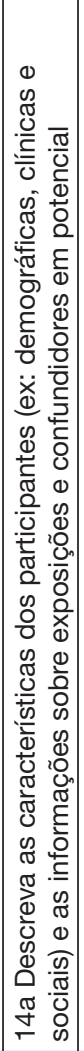 & 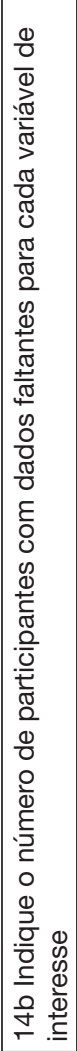 & 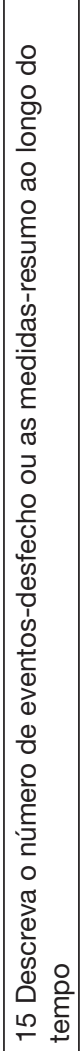 & 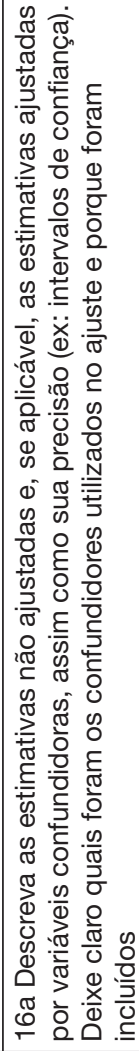 & 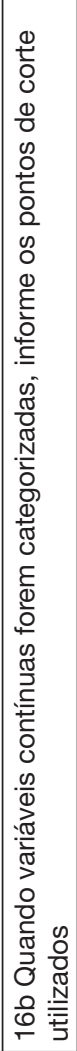 & 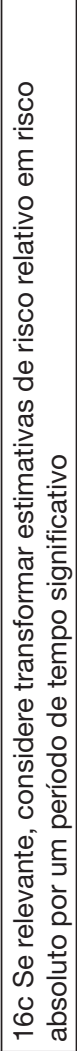 \\
\hline
\end{tabular}




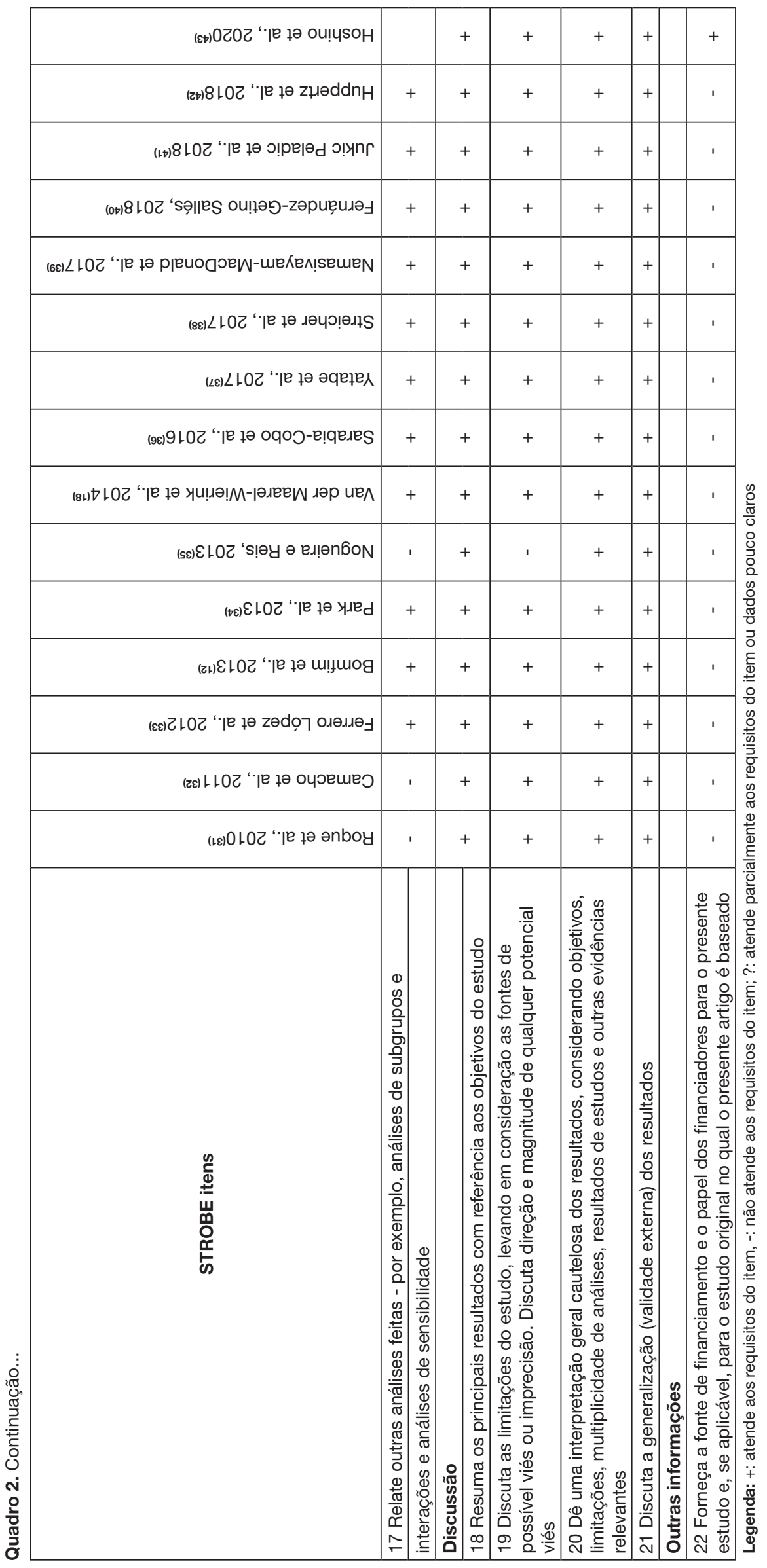




\section{DISCUSSÃO}

Esta revisão indicou ampla variabilidade da frequência de disfagia orofaríngea em idosos residentes em ILPI, além de abordagens metodológicas heterogêneas entre os estudos. Houve maior concentração de pesquisas em países europeus, nos quais a ocorrência de disfagia orofaríngea em idosos institucionalizados supera $60 \%{ }^{(33,36,44)}$. Estima-se que até 40 milhões de cidadãos da Europa, 16 milhões de americanos, e 8 milhões de japoneses necessitem de cuidados para disfagia ${ }^{(45)}$, números que devem aumentar ainda mais com o aumento do número de pessoas muito idosas, ou seja, aquelas com mais de 85 anos em países desenvolvidos e mais de 80 anos em países subdesenvolvidos ${ }^{(2)}$.

Em todos os artigos, a média de idade dos indivíduos foi acima de 80 anos. Em geral, o perfil de pessoas muito idosas é constituído por indivíduos que requerem maior assistência devido a presença de demência ${ }^{(46)}$, dependência e várias comorbidades que comprometem diversas atividades da vida diária, incluindo a alimentação ${ }^{(47)}$. Estudos já evidenciaram, nesses casos, a ausência da família, a dificuldade da família em cuidar, as relações familiares conflituosas aliadas à carência de renda e a falta de moradia como fatores associados ao aumento da necessidade desses idosos de um apoio a longo prazo de instituições de longa permanência ${ }^{(48,49)}$.

O perfil da amostra de todos os estudos incluídos foi dado pelo predomínio de pessoas do sexo feminino. O predomínio do sexo feminino entre indivíduos com 60 anos ou mais é relatado em diversos outros estudos e está atrelado ao que se chama de feminização da velhice, caracterizado pela maior proporção de mulheres na população idosa, especialmente em idades mais avançadas $^{(50-52)}$. Isso acontece em virtude de aspectos como melhor e mais frequente autocuidado com a saúde, menor exposição à morte por causas externas quando mais jovens, menor exposição a ambientes ocupacionais insalubres, cuidado mais rigoroso com a alimentação e conservação de estilo de vida menos prejudicial à saúde ${ }^{(53,54)}$.

Ao contrário do perfil de idade e sexo, as amostras apresentaram grande heterogeneidade tanto em relação à quantidade de idosos quanto ao número de ILIPI participantes. A menor amostra foi

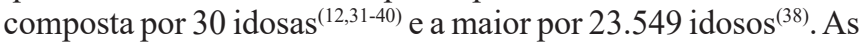
diferenças na composição do tamanho amostral nos diferentes estudos e localizações geográficas acentuam a dificuldade em determinar com precisão a frequência de disfagia orofaríngea em idosos institucionalizados, o que ficou evidente quando se observou as margens de erro das estimativas. Portanto, vale destacar a importância do desenho de cálculo amostral e do processo de amostragem para a obtenção de dados mais precisos e confiáveis. A realização de estudos de prevalência, ou seja, com amostras representativas e de base populacional, deve ser estimulada para se ter a real dimensão do problema na comunidade de idosos residentes em ILPI.

Os resultados revelaram uma compreensão heterogênea dos pesquisadores sobre a definição conceitual de disfagia orofaríngea. De forma geral, a maioria dos artigos reconheceu esse desfecho como uma alteração, dificuldade, desconforto, distúrbio, ou comprometimento da deglutição ${ }^{(12,19,32-37,39,41)}$. Apenas dois estudos mencionaram eficácia e/ou insegurança em suas definições ${ }^{(38,40)}$ e três não deixaram o conceito explícito ${ }^{(31,42,43)}$. Esse resultado indica que a carência de uma padronização conceitual é crítica e limita a obtenção de uma informação válida, precisa e confiável sobre a frequência da disfagia orofaríngea nas ILPI.
Os resultados desta revisão mostraram que a estimativa da frequência de disfagia orofaríngea em idosos institucionalizados possui ampla variação na literatura (5.4\% a 83.7\%), o que evidenciou a dificuldade em determinar esse dado na população estudada. Além de aspectos já mencionados como o tamanho amostral e o conceito de disfagia orofaríngea, outra característica que pode explicar essa grande amplitude da frequência é a utilização de instrumentos diferentes para determinar a presença do desfecho.

Nesta revisão, as frequências mais baixas foram observadas em pesquisas que utilizaram questionários e as mais altas quando foi realizada a avaliação clínica da deglutição. Os questionários são alternativas mais rápidas e baratas em estudos com amostras mais robustas nas quais o diagnóstico por meio de testes clínicos é menos viável e quando se tem como objetivo realizar um diagnóstico epidemiológico. Nesta revisão, percebeu-se o uso frequente de questionários em estudos com amostras maiores, $\mathrm{o}$ que resultou em estimativas mais baixas, porém, mais precisas se comparadas às obtidas por testes clínicos. É necessário ressaltar que até o momento não existem questionários com qualidade metodológica e propriedades psicométricas suficientes para realizar o rastreamento de disfagia orofaríngea em idosos ${ }^{(55)}$, embora já existam esforços nesse sentido, especialmente vislumbrando a aplicabilidade em estudos epidemiológicos ${ }^{(56)}$. Portanto, os questionários utilizados nos estudos incluídos nesta revisão possuem essa limitação e esse é um aspecto que deve ser ponderado ao analisar as estimativas de frequência encontradas.

Além disso, sabe-se que a população idosa aceita os transtornos de deglutição como um evento não tratável e natural do envelhecimento $^{(57)}$, o que acaba comprometendo o reconhecimento dos sintomas de disfagia orofaríngea e a confiabilidade da informação autorreferida. A ausência de questionários validados aliado a uma possível atitude de resignação da população diante do sintoma podem ajudar a explicar o resultado observado nesta revisão. Considerando esse cenário, percebe-se que há uma lacuna de estudos de prevalência que usem instrumentos adequados para o diagnóstico epidemiológico da disfagia orofaríngea em idosos. Recomenda-se, portanto, que os pesquisadores sejam criteriosos e optem pelo uso do instrumento com a melhor evidência de validade e confiabilidade possível ao planejarem suas pesquisas.

Quanto aos recursos clínicos para detectar disfagia orofaríngea em idosos, não há consenso sobre a utilização de um único instrumento ${ }^{(58)}$ e isso foi observado nesta revisão dada a diversidade encontrada. Além disso, nenhum estudo usou exames instrumentais para avaliação da disfagia orofaríngea. Sabe-se que a avaliação clínica da deglutição em algumas situações não é suficiente para detectar a presença da disfagia de modo preciso, haja vista que esta não é sensível, por exemplo, à detecção de resíduos em difícil localização, o que pode levar à aspiração tardia, sendo requeridos métodos instrumentais de avaliação ${ }^{(59)}$.

Contudo, a utilização de procedimentos instrumentais de diagnóstico nem sempre é acessível na prática diária, devido a restrições econômicas, limitação de profissionais e falta de equipamentos $^{(60)}$. Além disso, não é necessário ou viável realizar uma avaliação completa em todos os indivíduos ${ }^{(61)}$ e são por essas razões que os testes diagnósticos não instrumentais devem se preocupar em produzir resultados com a melhor acurácia possível.

A acurácia é uma propriedade de eficiência do procedimento diagnóstico e representa o grau de concordância entre o resultado do procedimento e o desfecho do melhor método disponível para determinar a presença ou ausência de uma condição de saúde ${ }^{(62-64)}$, que no caso da disfagia orofaríngea pode ser a videofluoroscopia da deglutição ou a videoendoscopia da deglutição ${ }^{(61)}$. A acurácia 
determina o poder do teste diagnóstico em discriminar quem tem e não tem a condição de saúde investigada ${ }^{(64)}$ e reflete as diversas relações entre os verdadeiros e falsos positivos e negativos por meio de indicadores como sensibilidade, especificidade, valor preditivo positivo e negativo, razão de verossimilhança positiva e negativa e curva ROC (receiver operator characteristic curve) ${ }^{(65)}$.

Os diferentes instrumentos de avaliação utilizados nos estudos incluídos nesta revisão integrativa também possuem acurácia distinta ou sequer investigada, o que interfere diretamente na confiabilidade e precisão das estimativas encontradas e reflete a dificuldade em determinar um instrumento de avaliação ou rastreamento que reúna evidências suficientes para ser implementado na prática.

Dessa forma, os resultados apresentados nesta revisão atestaram que embora a disfagia orofaríngea seja uma condição reconhecidamente presente nos idosos residentes em ILPI, os estudos disponíveis até o momento em relação à frequência dessa condição nessa população são metodologicamente muito distintos e não permitem estimar o dado com precisão. Além disso, é necessário refletir sobre a padronização conceitual sobre o desfecho, a necessidade de estudos epidemiológicos e o desenvolvimento de instrumentos viáveis que produzam resultados válidos e confiáveis para o diagnóstico de disfagia orofaríngea na população idosa institucionalizada.

\section{CONCLUSÃO}

A frequência de disfagia orofaríngea em idosos institucionalizados variou entre $5.4 \%$ e $83.7 \%$, com discrepâncias metodológicas em relação ao tamanho da amostra, conceito de disfagia orofaríngea e instrumentos de avaliação utilizados para o diagnóstico.

\section{REFERENCIAS}

1. United Nations. World population prospects 2019 highlights. New York: United Nations; 2019.

2. United Nations. World population prospects 2019 volume II: demographic profiles. New York: United Nations; 2019.

3. IBGE: Instituto Brasileiro de Geografia e Estatística. 2010 census [Internet]. 2010 [citado em 2020 Maio 28]. Disponível em: https://censo2010.ibge. gov.br/sobre-censo.html

4. Sander M, Oxlund B, Jespersen A, Krasnik A, Mortensen EL, Westendorp $\mathrm{RG}$, et al. The challenges of human population ageing. Age Ageing. 2015;44(2):185-7. http://dx.doi.org/10.1093/ageing/afu189. PMid:25452294.

5. Chen PH, Golub JS, Hapner ER, Johns MM 3rd. Prevalence of perceived dysphagia and quality-of-life impairment in a geriatric population. Dysphagia. 2009;24(1):1-6. http://dx.doi.org/10.1007/s00455-008-9156-1. PMid: 18368451.

6. Langmore SE, Skarupski KA, Park PS, Fries BE. Predictors of aspiration pneumonia in nursing home residents. Dysphagia. 2002;17(4):298-307. http://dx.doi.org/10.1007/s00455-002-0072-5. PMid:12355145.

7. Brodsky MB, McNeil MR, Martin-Harris B, Palmer CV, Grayhack JP, Abbott KV. Effects of divided attention on swallowing in healthy participants. Dysphagia. 2012;27(3):307-17. http://dx.doi.org/10.1007/ s00455-011-9367-8. PMid:21892783.

8. Logemann JA, Curro FA, Pauloski B, Gensler G. Aging effects on oropharyngeal swallow and the role of dental care in oropharyngeal dysphagia. Oral Dis. 2013;19(8):733-7. http://dx.doi.org/10.1111/odi.12104. PMid:23574512.

9. Rofes L, Arreola V, Romea M, Palomera E, Almirall J, Cabré M, et al. Pathophysiology of oropharyngeal dysphagia in the frail elderly. Neurogastroenterol Motil. 2010;22(8):851-8, e230. http://dx.doi. org/10.1111/j.1365-2982.2010.01521.x. PMid:20529208.

10. Cabré M, Serra-Prat M, Force L, Almirall J, Palomera E, Clavé P. Oropharyngeal dysphagia is a risk factor for readmission for pneumonia in the very elderly persons: observational prospective study. J Gerontol A Biol Sci Med Sci. 2014;69(3):330-7. http://dx.doi.org/10.1093/gerona/ glt099. PMid:23833199.

11. Serra-Prat M, Palomera M, Gomez C, Sar-Shalom D, Saiz A, Montoya $\mathrm{JG}$, et al. Oropharyngeal dysphagia as a risk factor for malnutrition and lower respiratory tract infection in independently living older persons: a population-based prospective study. Age Ageing. 2012;41(3):376-81. http://dx.doi.org/10.1093/ageing/afs006. PMid:22311895.

12. Bomfim FMS, Chiari BM, Roque FP. Factors associated to suggestive signs of oropharyngeal dysphagia in institutionalized elderly women. CoDAS. 2013;25(2):154-63. http://dx.doi.org/10.1590/S2317-17822013000200011. PMid:24408245.

13. Aslam M, Vaezi MF. Dysphagia in the elderly. Gastroenterol Hepatol. 2013;9(12):784-95. PMid:24772045.

14. Furkim AM. Disfagia orofaríngea neurogênica. In: Marchesan IC, editor Fundamentos em fonoaudiologia: aspectos clínicos da motricidade oral. Rio de Janeiro: Guanabara Koogan. 2005. p. 121-32.

15. Langmore SE, Skarupski KA, Park PS, Fries BE. Predictors of aspiration pneumonia in nursing home residents. Dysphagia. 2002;17(4):298-307. http://dx.doi.org/10.1007/s00455-002-0072-5. PMid:12355145.

16. Santoro PP. Disfagia orofaríngea: panorama atual, epidemiologia, opções terapêuticas e perspectivas futuras. Rev CEFAC. 2008;10(2). http://dx.doi. org/10.1590/S1516-18462008000200002.

17. Marik PE, Kaplan D. Aspiration pneumonia and dysphagia in the elderly. Chest. 2003;124(1):328-36. http://dx.doi.org/10.1378/chest.124.1.328. PMid:12853541.

18. Van der Maarel-Wierink CD, Meijers JM, De Visschere LM, de Baat C, Halfens RJ, Schols JM. Subjective dysphagia in older care home residents: a crosssectional, multi-centre point prevalence measurement. Int J Nurs Stud. 2014:51(6):875-81. http://dx.doi.org/10.1016/j.ijnurstu.2013.10.016. PMid:24238894.

19. Roy N, Stemple J, Merrill RM, Thomas L. Dysphagia in the elderly: preliminary evidence of prevalence, risk factors, and socioemotional effects. Ann Otol Rhinol Laryngol. 2007;116(11):858-65. http://dx.doi. org/10.1177/000348940711601112. PMid:18074673.

20. Van der Maarel-Wierink CD, Vanobbergen JN, Bronkhorst EM, Schols $\mathrm{JM}$, de Baat C. Risk factors for aspiration pneumonia in frail older people: a systematic literature review. J Am Med Dir Assoc. 2011;12(5):344-54. http://dx.doi.org/10.1016/j.jamda.2010.12.099. PMid:21450240.

21. Palmu AA, Saukkoriipi A, Snellman M, Jokinen J, Torkko P, Ziegler T, et al Incidence and etiology of community-acquired pneumonia in the elderly in a prospective population-based study. Scand J Infect Dis. 2014;46(4):250-9. http://dx.doi.org/10.3109/00365548.2013.876509. PMid:24475952.

22. Sue Eisenstadt E. Dysphagia and aspiration pneumonia in older adults. J Am Acad Nurse Pract. 2010;22(1):17-22. http://dx.doi.org/10.1111/j.17457599.2009.00470.x. PMid:20074192.

23. Sakai K, Sakuma K. Sarcopenic dysphagia as a new concept. In: Dionyssiotis Y, editor. Frailty sarcopenia: onset, development and clinical challenges. London: IntechOpen; 2017. http://dx.doi.org/10.5772/intechopen.68791.

24. Fujishima I, Fujiu-Kurachi M, Arai H, Hyodo M, Kagaya H, Maeda K, et al Sarcopenia and dysphagia: position paper by four professional organizations. Geriatr Gerontol Int. 2019;19(2):91-7. http://dx.doi.org/10.1111/ggi.13591. PMid:30628181.

25. Steele CM, Greenwood C, Ens I, Robertson C, Seidman-Carlson R. Mealtime difficulties in a home for the aged: not just dysphagia. Dysphagia. 1997;12(1):43-50. http://dx.doi.org/10.1007/PL00009517. PMid:8997832.

26. Almirall J, Cabré M, Clavé P. Neumonía aspirativa. Med Clin. 2007;129(11):42432. http://dx.doi.org/10.1157/13110467. PMid:17927938.

27. Shanley C, O'Loughlin G. Dysphagia among nursing home residents: an assessment and management protocol. J Gerontol Nurs. 2000;26(8):35-48. http://dx.doi.org/10.3928/0098-9134-20000801-09. PMid:11276612.

28. Mendes KDS, Silveira RCCP, Galvão CM. Revisão integrativa: método de pesquisa para a incorporação de evidências na saúde e na enfermagem. Texto Contexto Enferm. 2008;17(4):758-64. http://dx.doi.org/10.1590/ S0104-07072008000400018.

29. von Elm E, Altman DG, Egger M, Pocock SJ, Gøtzsche PC, Vandenbroucke JP. The Strengthening the Reporting of Observational Studies in Epidemiology (STROBE) statement: guidelines for reporting observational studies. Int J Surg. 2014;12(12):1495-9. http://dx.doi.org/10.1016/j.ijsu.2014.07.013. PMid:25046131. 
30. Malta M, Cardoso LO, Bastos FI, Magnanini MMF, Silva CMFP. STROBE Initiative: grants paragraph communication observational studies. Rev Saude Publica. 2010;44(3):559-65. http://dx.doi.org/10.1590/S003489102010000300021. PMid:20549022.

31. Roque FP, Bomfim FMS, Chiari BM. Descrição da dinâmica de alimentação de idosas institucionalizadas. Rev Soc Bras Fonoaudiol. 2010;15(2):25663. http://dx.doi.org/10.1590/S1516-80342010000200018.

32. Camacho MJT, Perozo MV, Sabaté AP, Acosta MEG. Disfagia en ancianos que viven en residencias geriátricas de Barcelona. Gerokomos. 2011;22(1):20-4.

33. Ferrero López MI, García Gollarte JF, Botella Trelis JJ, Juan Vidal O. Detección de disfagia en mayores institucionalizados. Rev Esp Geriatr Gerontol. 2012;47(4):143-7. http://dx.doi.org/10.1016/j.regg.2011.09.004. PMid:22264749.

34. Park Y, Han HR, Oh BM, Lee J, Park JA, Yu SJ, et al. Prevalence and associated factors of dysphagia in nursing home residents. Geriatr Nurs. 2013;34(3):212-7. http://dx.doi.org/10.1016/j.gerinurse.2013.02.014. PMid:23528180

35. Nogueira D, Reis E. Swallowing disorders in nursing home residents: how can the problem be explained? Clin Interv Aging. 2013;8:8. http://dx.doi. org/10.2147/CIA.S39452. PMid:23449951.

36. Sarabia-Cobo CM, Pérez V, de Lorena P, Domínguez E, Hermosilla C, Nuñez MJ, et al. The incidence and prognostic implications of dysphagia in elderly patients institutionalized: a multicenter study in Spain. Appl Nurs Res. 2016;30:e6-9. http://dx.doi.org/10.1016/j.apnr.2015.07.001. PMid:26235494.

37. Yatabe N, Takeuchi K, Izumi M, Furuta M, Takeshita T, Shibata Y, et al. Decreased cognitive function is associated with dysphagia risk in nursing home older residents. Gerodontology. 2018;35(4):1-6. http://dx.doi. org/10.1111/ger.12366. PMid:30028036.

38. Streicher M, Wirth R, Schindler K, Sieber CC, Hiesmayr M, Volkert D. Dysphagia in nursing homes: results from the NutritionDay Project. J Am Med Dir Assoc. 2018;19(2):141-147.e2. http://dx.doi.org/10.1016/j. jamda.2017.08.015. PMid:29030310.

39. Namasivayam-MacDonald AM, Morrison JM, Steele CM, Keller H. How swallow pressures and dysphagia affect malnutrition and mealtime outcomes in long-term care. Dysphagia. 2017;32(6):785-96. http://dx.doi. org/10.1007/s00455-017-9825-z. PMid:28733775.

40. Fernández-Getino Sallés C. Prevalencia de disfagia orofaríngea en ancianos institucionalizados y su relación con síndromes geriátricos. Rev Logop Fon Audiol. 2018;38(2):69-76. http://dx.doi.org/10.1016/j.rlfa.2017.09.001.

41. Jukic Peladic N, Orlandoni P, Dell'Aquila G, Carrieri B, Eusebi P, Landi $\mathrm{F}$, et al. Dysphagia in nursing home residents: management and outcomes. J Am Med Dir Assoc. 2019;20(2):147-51. http://dx.doi.org/10.1016/j. jamda.2018.07.023. PMid:30249360.

42. Huppertz VAL, Halfens RJG, Van Hel Voort A, De Groot LCPGM, Baijens LWJ, Schols JMGA. Association between oropharyngeal dysphagia and malnutrition in dutch nursing home residents: results of the national prevalence measurement of quality of care. J Nutr Health Aging. 2018;22(10):1246-52. http://dx.doi.org/10.1007/s12603-018-1103-8. PMid:30498833.

43. Hoshino D, Watanabe Y, Edahiro A, Kugimiya Y, Igarashi K, Motokawa $\mathrm{K}$, et al. Association between simple evaluation of eating and swallowing function and mortality among patients with advanced dementia in nursing homes: 1-year prospective cohort study. Arch Gerontol Geriatr. 2020;87:103969. http://dx.doi.org/10.1016/j.archger.2019.103969. PMid:31783307.

44. Baijens LW, Clavé P, Cras P, Ekberg O, Forster A, Kolb GF, et al. European Society for Swallowing Disorders - European Union Geriatric Medicine Society white paper: oropharyngeal dysphagia as a geriatric syndrome. Clin Interv Aging. 2016;11:1403-28. http://dx.doi.org/10.2147/CIA.S107750. PMid:27785002.

45. Dziewas R, Beck AM, Clave P, Hamdy S, Heppner HJ, Langmore SE, et al. Recognizing the importance of dysphagia: stumbling blocks and stepping stones in the twenty-first century. Dysphagia. 2017;32(1):78-82. http:// dx.doi.org/10.1007/s00455-016-9746-2. PMid:27571768.

46. Irles Rocamora JA, Sánchez-Duque MJ, de Valle Galindo PB, Bernal López E, Fernández Palacín A, Almeida González C, et al. A prevalence study of dysphagia and intervention with dietary counselling in nursing home from Seville. Nutr Hosp. 2009;24(4):498-503. PMid:19721932.

47. Simmons SF, Schnelle JF. Feeding assistance needs of long-stay nursing home residents and staff time to provide care. J Am Geriatr Soc. 2006;54(6):91924. http://dx.doi.org/10.1111/j.1532-5415.2006.00812.x. PMid:16776786.

48. OMS: Organização Mundial de Saúde. Relatório mundial de envelhecimento e saúde. OMS; 2015.
49. Souza IAL, Massi G. Rev. A saúde fonoaudiológica a partir do discurso do idoso institucionalizado. CEFAC. 2015;17(1):300-7. http://dx.doi. org/10.1590/1982-0216201519413.

50. Drummond A, Alves ED. Perfil socioeconômico e demográfico e a capacidade funcional de idosos atendidos pela Estratégia Saúde da Família de Paranoá, Distrito Federal. Rev Bras Geriatr Gerontol. 2013;16(4):72738. http://dx.doi.org/10.1590/S1809-98232013000400007.

51. Confortin SC, Antes DL, Pessini J, Schneider IJC, d'Orsi E, Barbosa AR. Comparação do perfil socioeconômico e condições de saúde de idosos residentes em áreas predominantemente rural e urbana da Grande Florianópolis, Sul do Brasil. Cad Saude Colet. 2016;24(3):330-8. http:// dx.doi.org/10.1590/1414-462x201600030034.

52. Sousa NFS, Lima MG, Cesar CLG, Barros MBA. Envelhecimento ativo: prevalência e diferenças de gênero e idade em estudo de base populacional. Cad Saude Publica. 2018;34(11):e00173317. http://dx.doi.org/10.1590/0102311x00173317. PMid:30484561.

53. Silva SPC, Menandro MCS. As representações sociais da saúde e de seus cuidados para homens e mulheres idosos. Saude Soc. 2014;23(2):626-40. http://dx.doi.org/10.1590/S0104-12902014000200022.

54. Oliveira AC, Santos AA, Pavirini SCI. Relação entre sintomas depressivos e a funcionalidade familiar de idosos institucionalizados. Rev Esc Enferm USP. 2014;48(1):66-72. PMid:24676110.

55. Magalhães HV Jr, Pernambuco LA, Lima KC, Ferreira MAF. Screening for oropharyngeal dysphagia in older adults: A systematic review of selfreported questionnaires. Gerodontology. 2018;35(3):162-9. http://dx.doi. org/10.1111/ger.12333. PMid:29611876.

56. Magalhães HV Jr, Pernambuco LA, Cavalcanti RVA, Lima KC, Ferreira MAF. Validity evidence of an epidemiological oropharyngeal dysphagia screening questionnaire for older adults. Clinics. 2020;75:e1425.; published online Jan 10, 2020. http://dx.doi.org/10.6061/clinics/2020/e1425. PMid:31939561.

57. Ekberg O, Hamdy S, Woisard V, Wuttge-Hannig A, Ortega P. Social and psychological burden of dysphagia: its impact on diagnosis and treatment Dysphagia. 2002;17(2):139-46. http://dx.doi.org/10.1007/s00455-0010113-5. PMid:11956839.

58. Leder SB, Suiter DM, Warner HL. Answering orientation questions and following single-step verbal commands: effect on aspiration status. Dysphagia. 2009;24(3):290-5. http://dx.doi.org/10.1007/s00455-008-9204-x. PMid:19263106.

59. Sordi M, Mourão LF, Silva AA, Flosi LCL. Importância da interdisciplinaridade na avaliação das disfagias: avaliação clínica e videofluoroscópica da deglutição. Rev Bras Otorrinolaringol. 2009;75(6):776-87.

60. Hammond CAS, Goldstein LB. Cough and aspiration of food and liquids due to oral-pharyngeal dysphagia: ACCP evidence-based clinical practice guidelines. Chest. 2006;129(1, Supl):154S-68S. http://dx.doi.org/10.1378/ chest.129.1_suppl.154S. PMid:16428705.

61. Suiter D. Dysphagia Screening: challenges and controversies. Perspect ASHA Spec Interest Groups. 2018;3(13):82-8. http://dx.doi.org/10.1044/ persp3.SIG13.82.

62. Schünemann HJ, Oxman AD, Brozek J, Glasziou P, Jaeschke R, Vist $\mathrm{GE}$, et al. Grading quality of evidence and strength of recommendations for diagnostic tests and strategies. BMJ. 2008;336(7653):1106-10. http:/ dx.doi.org/10.1136/bmj.39500.677199.AE. PMid:18483053.

63. Rutjes AW, Reitsma JB, Coomarasamy A, Khan KS, Bossuyt PM. Evaluation of diagnostic tests when there is no gold standard: a review of methods. Health Technol Assess. 2007;11(50):iii-51. http://dx.doi.org/10.3310/ hta11500. PMid:18021577.

64. Umemneku Chikere CM, Wilson K, Graziadio S, Vale L, Allen AJ. Diagnostic test evaluation methodology: a systematic review of methods employed to evaluate diagnostic tests in the absence of gold standard: an update. PLoS One. 2019;14(10):e0223832. http://dx.doi.org/10.1371/ journal.pone.0223832. PMid:31603953.

65. Pernambuco L, Espelt A, Magalhães HV, Lima KC. Recommendations for elaboration, transcultural adaptation and validation process of tests in Speech, Hearing and Language Pathology. CoDAS. 2017;29(3):e20160217.PMid:28614460.

\section{Contribuição dos autores}

JSX contribuiu com a coleta, análise dos dados, redação e revisão final do artigo, ACBG e LCPT contribuiu com a coleta e análise dos dados; LP contribuiu com a concepção, orientação, análise dos dados, redação e revisão final do artigo. 
Apêndice A. Estratégias de busca utilizadas nas bases de dados

\begin{tabular}{|c|c|}
\hline Base de dados & Descritores \\
\hline Pubmed/Medline & $\begin{array}{l}\text { ((((“"nursing homes”[MeSH Terms]) OR (“nursing home"[Title/Abstract]])) OR (“long term care facilities”[Title/Abstract]])) OR } \\
\text { (“"home for the aged”[Title/Abstract])) AND (((((“deglutition”[MeSH Terms]) OR (deglutition disorder[MeSH Terms])) OR } \\
\text { (dysphagia[Title/Abstract])) OR (swallowing[Title/Abstract])) OR (deglutition[Title/Abstract])) }\end{array}$ \\
\hline Web of Science & $\begin{array}{l}\text { TS=((dysphagia OR (deglutition OR swallow*)) AND ("nursing home" OR ("nursing homLes" OR "long term care facility" } \\
\text { OR "long term care facilities"))) }\end{array}$ \\
\hline Scopus & $\begin{array}{l}\text { TITLE-ABS-KEY((dysphagia OR (deglutition OR swallow*)) AND (“nursing home" OR (“nursing homes" OR "long term } \\
\text { care facility" OR "long term care facilities"))) }\end{array}$ \\
\hline LILACS & “DISFAGIA” [Palavras] and “INSTITUICAO DE LONGA PERMANENCIA PARA IDOSOS” [Palavras] \\
\hline SciELO & "DISFAGIA" [Palavras] and "INSTITUICAO DE LONGA PERMANENCIA PARA IDOSOS” [Palavras] \\
\hline
\end{tabular}

